\title{
El acogimiento en familia ajena en España. Una evaluación de la práctica y sus resultados
}

\author{
MÓNica López ${ }^{1}$, CARMen MONTSERRAT ${ }^{2}$, JORGE F. Del VALLE ${ }^{1}$ \\ Y AMAIA BRAVO ${ }^{1}$ \\ ${ }^{1}$ Universidad de Oviedo; ${ }^{2}$ Universidad de Girona
}

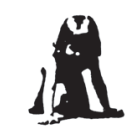

Resumen

El acogimiento familiar ha de ser la medida prioritaria para los casos de menores que deben ser separados de su familia por motivos de protección. En España es una alternativa que cuenta tan sólo con veinte años de existencia y hasta la fecha no existen prácticamente datos acerca del grado de su implantación y sus características cuando se trata de acogimiento en familia ajena, mientras que son varios los trabajos que han estudiado el acogimiento en familia extensa. Este artículo presenta por primera vez en la literatura científica los datos más relevantes que permiten caracterizar la práctica del acogimiento en familia ajena en España, mediante el estudio de una muestra de seis comunidades autónomas bien representativas, con un total de 357 casos. El artículo presenta los perfiles de los niños, las familias biológicas y acogedoras, el proceso y algunos resultados sobre una submuestra de casos cerrados $(n=179)$. Se analizarán algunas características especialmente importantes como la larga estancia y la estabilidad de estos acogimientos, que los diferencia de los realizados en otros países.

Palabras clave: Acogimiento familiar, protección infantil, acogimiento en familia ajena, evaluación de programas.

\section{Non-relative family foster care in Spain: Practice and outcomes assessment}

\begin{abstract}
Family foster care must be given priority in out-of-home placement decisions involving minors who need to be separated from their family for their own protection. In Spain, this alternative has only been possible for the past twenty years. To date there are practically no data available about the extent of its implementation and characteristics in the case of nonrelative foster care, though there are several research studies about kinship care. The paper discusses the most relevant data in order to depict -for the first time in scientific literature - the practice of non-relative foster care in Spain. For this purpose, we study a sample of 357 cases encompassing six representative Autonomous Communities. We also presents children's profiles, birth and foster families' characteristics, information about the fostering process and some results based on a subsample of closed cases $(n=179)$. Some important features such as the long stay periods and stability of placements, which differentiates them from foster care practice in other countries, are also analysed.
\end{abstract}

Keywords: Foster care, child care, non-relative foster care, programme evaluation.

Agradecimientos: Esta investigación ha sido financiada por el Ministerio de Trabajo y Asuntos Sociales mediante el proyecto CN-06-042 realizado con la Universidad de Oviedo.

La primera autora disfruta de una beca predoctoral del Programa Severo Ochoa de la Fundación para el Fomento en Asturias de la Investigación Científica Aplicada y la Tecnología (FICYT).

Correspondencia con los autores: Jorge F. del Valle. Facultad de Psicología. Plaza Feijoo s/n. 33003 Oviedo. E-mail: jvalle@uniovi.es 
Los servicios sociales para la infancia y la familia en España tienen una corta trayectoria como sistema vinculado al concepto de Estado del Bienestar. Hasta los años ochenta la protección de los menores de edad estaba en manos de una serie de patronatos e instituciones que mediante un modelo de beneficencia ejercían esta protección con la medida de institucionalización. La respuesta para los niños y jóvenes desprotegidos era su separación del domicilio familiar y su ingreso en instituciones donde permanecían muchos años y en ocasiones toda su infancia (Del Valle, Álvarez-Baz y Bravo, 2003).

Las reformas legislativas de los años ochenta y noventa establecieron una ruptura con esta práctica centrada en el "rescate" de los menores de edad desprotegidos. En su lugar, se instauró un modelo que dio prioridad al trabajo con las familias como primera intervención, con la finalidad de preservar la convivencia cuando fuera posible. Desde entonces, en los casos en que la separación sea necesaria para garantizar el bienestar de los niños y niñas, se deberá buscar, siempre que sea posible, un contexto familiar alternativo y no un establecimiento residencial.

La búsqueda de este nuevo contexto familiar, cuando el propio no puede cubrir adecuadamente las necesidades de los niños, se promovía ya en 1987 con la reforma del Código Civil que introducía la medida de acogimiento familiar en España, además de facilitar los procesos de adopción (Ley 21/1987). Así pues, en nuestro país el acogimiento familiar tiene una regulación que cumple en la actualidad poco más de veinte años, mientras que en otros países cuenta con más de medio siglo de historia. Este retraso ha sido uno de los motivos de que nuestro marco jurídico haya enfatizado la necesidad de dar un gran impulso a esta medida.

Sin embargo, el acogimiento familiar en España ha contado con muy escasa investigación y evaluación, de modo que difícilmente se pueden extraer conclusiones acerca de su grado de implantación y mucho menos de sus resultados. En una revisión reciente sobre el tema (Palacios y Amorós, 2006), no se encontraron más de media docena de artículos de investigación sobre acogimiento familiar realizados en España.

Por otro lado, dada la importancia que el acogimiento en familia extensa tiene en nuestro país, donde supone el $85 \%$ frente a un $15 \%$ en familia ajena (Del Valle y Bravo, 2003), en los últimos años sabemos un poco más acerca de los perfiles y procesos del acogimiento con los familiares que cuando se produce con otras personas (Bernedo, Fuentes y Fernández, 2008; Del Valle, Álvarez-Baz y Bravo, 2002; Lumbreras, Fuentes y Bernedo, 2005; Molero, Mora, Albiñana y Gimeno, 2006; Montserrat, 2007; Montserrat y Casas, 2006). Otra limitación es la falta de estadísticas nacionales, ya que a pesar de que existe un Boletín Estadístico de Datos Básicos de Protección a la Infancia (Ministerio de Trabajo y Asuntos Sociales, 2007) únicamente se contabilizan el número de las medidas y actos administrativos y judiciales, pero no indican nada sobre cuestiones tan elementales como edades, sexo, duración, perfiles de las familias, etc.

Respecto a estudios que engloban tanto acogimiento en familia ajena como extensa, sobresale la evaluación del programa de Familias Canguro (Amorós, Palacios, Fuentes, León y Mesas, 2003) mediante una investigación longitudinal realizada en nueve comunidades autónomas. Estos autores, de las Universidades de Barcelona y Sevilla, han contribuido notablemente al desarrollo del acogimiento en España, incluyendo la construcción de materiales e instrumentos para los profesionales y las familias (Amorós, Palacios, Fuentes, León y Mesas, 2002, por ejemplo). Por otra parte, ha constituido un punto de referencia el informe elaborado por Del Valle y Bravo (2003) sobre la situación del acogimiento familiar en España, donde por vez primera se presentan datos descriptivos de la práctica en todas las comunidades autónomas. Recientemente, Palacios y Jiménez (2007) publicaron los resultados de un estudio sobre acogimiento familiar - en ajena y extensa- en Andalucía, donde se presentaban los principales datos y conclusiones referidos a los progenitores, acogedores, así como niños y niñas acogidos. Este estudio aporta información detallada sobre las características de los acogedores y el hogar de acogida, la estimulación que ofrecen al niño o niña, su nivel de estrés como acogedores, así como 
sus fuentes de apoyo social. Respecto a los niños y niñas acogidos, merece la pena resaltar el valor de esta investigación al indagar en aspectos tales como su autoestima y sus relaciones personales.

Estos trabajos concluyen que el acogimiento familiar cumple una función protectora muy importante, y que, en general, se cubren las necesidades tanto afectivas como materiales de los acogidos. Sin embargo, apuntan también la necesidad de implementar mejoras en cuanto a la formación, apoyo y seguimiento, para paliar ciertos aspectos de vulnerabilidad en los acogimientos familiares. Una limitación general es que, al estudiar los casos en activo, no suelen aportar datos sobre finalización, rupturas ni resultados en general.

En el panorama internacional, los estudios referidos al ámbito de los acogimientos llevan décadas de evolución y no es tarea fácil su selección. Sin embargo, en los últimos años el equipo de la Universidad de York ha realizado un importante conjunto de aportaciones y revisiones al tema del acogimiento familiar en el Reino Unido (Sinclair, 2005; Sinclair, Baker, Wilson y Gibbs, 2005; Sinclair, Gibbs y Wilson, 2004). Una importante conclusión general es que el acogimiento familiar en este contexto tiene dificultades para dar continuidad en la atención a estos menores, encontrándose un buen número de cambios de una familia a otra en estos procesos. Este aspecto ha sido subrayado también por Ward, Skuse y Munro (2005) en el estudio de acogimientos con niños más pequeños y se encuentra reflejado igualmente en trabajos realizados en Estados Unidos (Berrick, Needell, Barth y Jonhson-Reid, 1998; Frame, Berrick y Brodowsky, 2000) donde se señala que la ruptura de un acogimiento familiar supone casi siempre intentar otro nuevo. Este porcentaje de ruptura varía según una revisión realizada sobre el tema (Sallnäs, Vinnerljung y Westermark, 2004) entre el 30\% y el 37\% de los casos, encontrándose diferencias entre los distintos tipos de acogimiento. De entre los múltiples motivos de fracaso destacan los referidos a los problemas de conducta de los acogidos, o la enfermedad o fallecimiento de los acogedores (Vanderfaeillie, Van Holen y Coussens, 2008). Otro aspecto señalado es que cuando los jóvenes alcanzan la mayoría de edad, acostumbran a dejar la familia de acogida y los acogedores suelen realizar un nuevo acogimiento (Sinclair et al., 2005).

Este artículo se centrará en los acogimientos en familia ajena con el objetivo de describir los perfiles básicos de los protagonistas (niños y niñas, familias biológicas y familias acogedoras), procesos (procedimiento, existencia de visitas, etcétera) y resultados (motivo de finalización y situación posterior). Aunque el artículo es fundamentalmente descriptivo, su valor estriba en que es la primera vez que en España se presentan datos que permiten caracterizar el tipo de acogimiento familiar que se realiza con familia ajena, así como averiguar lo que ocurre en términos de resultados, al finalizar los acogimientos.

\section{Metodología}

\section{Muestra}

A través de seis comunidades autónomas se obtuvo una muestra, estratificada en proporción aproximada al número de acogimientos que realiza cada una de ellas, de 357 casos de acogimiento familiar realizado con personas sin relación de parentesco (Madrid: 115; Cataluña: 88; Comunidad Valenciana: 69; Galicia: 45; Castilla-León: 20 y Andalucía: 20). En el caso de Andalucía el tamaño muestral que hubiera correspondido sería mayor, pero diferentes problemas en la recogida de datos obligaron a reducir su tamaño.

Estas comunidades fueron elegidas por ser algunas de las que mayor número de acogimientos realizaban, según el estudio de Del Valle y Bravo (2003). Es importante destacar que aunque se trata solamente de seis comunidades autónomas, las aquí seleccionadas aportan el $75 \%$ de todos los acogimientos, según ese estudio, por lo que se puede considerar una muestra bastante representativa. Para poder evaluar resultados, se inclu- 
yó el criterio de que aproximadamente la mitad de los casos seleccionados hubieran sido cerrados durante los cinco años anteriores, obteniendo así una submuestra de 179 casos cerrados.

\section{Recogida de datos y variables}

Se elaboró una hoja de recogida de datos estructurada en categorías referidas a variables del perfil de los protagonistas, proceso de acogimiento y, para los casos cerrados, resultados de la intervención. Esta hoja fue completada por cinco colaboradores que trabajaron en cada comunidad autónoma para recabar la información mediante la revisión de expedientes y la realización de entrevistas con los profesionales responsables del caso y los de los programas de acogimiento. Los casos se seleccionaron aleatoriamente y toda la información se pudo recoger de forma anónima, respetando el derecho a la protección de datos personales.

Aunque se recogió un volumen de información muy extenso, los datos que se presentan en este artículo han sido agrupados de la siguiente forma:

(a) Perfil de los niños y niñas acogidos, las familias biológicas y los acogedores, fundamentalmente en cuanto a variables sociodemográficas;

(b) Proceso del acogimiento: situación previa, plan de caso, intervención judicial, visitas, lugar de las visitas;

(c) Resultados (para casos cerrados): duración, motivo de cierre, situación final.

Un aspecto especialmente importante es que este trabajo no utilizará la distinción entre simple y permanente, que como es sabido viene establecida por la legislación nacional. En el trabajo de Del Valle y Bravo (2003) se demostró que esta categoría tiene un uso completamente diferente según la comunidad de que se trate, hasta el punto de que en algunas, como Cataluña o Asturias, solamente se utilizaba el tipo simple, y en otras, como el caso de Madrid, más del $90 \%$ son permanentes. Esta situación se ratifica en nuestra muestra y por tanto, a pesar del pleno sentido que tendría diferenciar estos tipos desde un punto de vista teórico, no es pertinente hacerlo en las actuales circunstancias.

\section{Resultados}

\section{Perfil de los niños y niñas acogidos, las familias biológicas y los acogedores}

La proporción de niños $(50,1 \%)$ y niñas que componen la muestra resultó muy parecida (en la Tabla I se muestran los perfiles de todos los intervinientes en el proceso). Se distinguió su edad en dos momentos: el de realizar el acogimiento y el de apertura de su caso de protección. La media de la edad al inicio del acogimiento fue de cerca de siete años, iniciándose con mayor frecuencia en la etapa 4-8 años, aunque de modo muy similar a la etapa $0-3$, para descender a medida que avanzan las edades, con poco más de un $11 \%$ de casos acogidos con más de 13 años. Con respecto a la media de edad de apertura del caso de protección, esta fue de 4,5 años, lo que nos indica un periodo de espera de 2,5 años entre el inicio de la protección y la incorporación a un acogimiento familiar. La proporción de niños con alguna minusvalía diagnosticada fue del 8,1\%. Son pocos los casos de niños o niñas de otras nacionalidades $(7,8 \%)$ y como grupo étnico sólo apareció con cierta importancia el de niños y niñas de familias gitanas $(5,9 \%)$.

El estudio de las características de las familias de origen se hizo sobre la base del número de familias $(N=344)$ y no de niños $(N=357)$, ya que había algunos grupos de hermanos. Se encontró un porcentaje de fallecimientos del padre del 16,8\% y en menor grado de la madre $(6 \%)$. Destaca también el alto grado de rupturas familiares, con un $38,8 \%$ de separaciones o divorcios y la presencia de un $22 \%$ de madres solteras. La media de hijos e hijas es amplia para lo que es normativo en la sociedad española, ya que se obtuvo un promedio de casi tres hijos. En cuanto a problemas psicosociales destaca- 


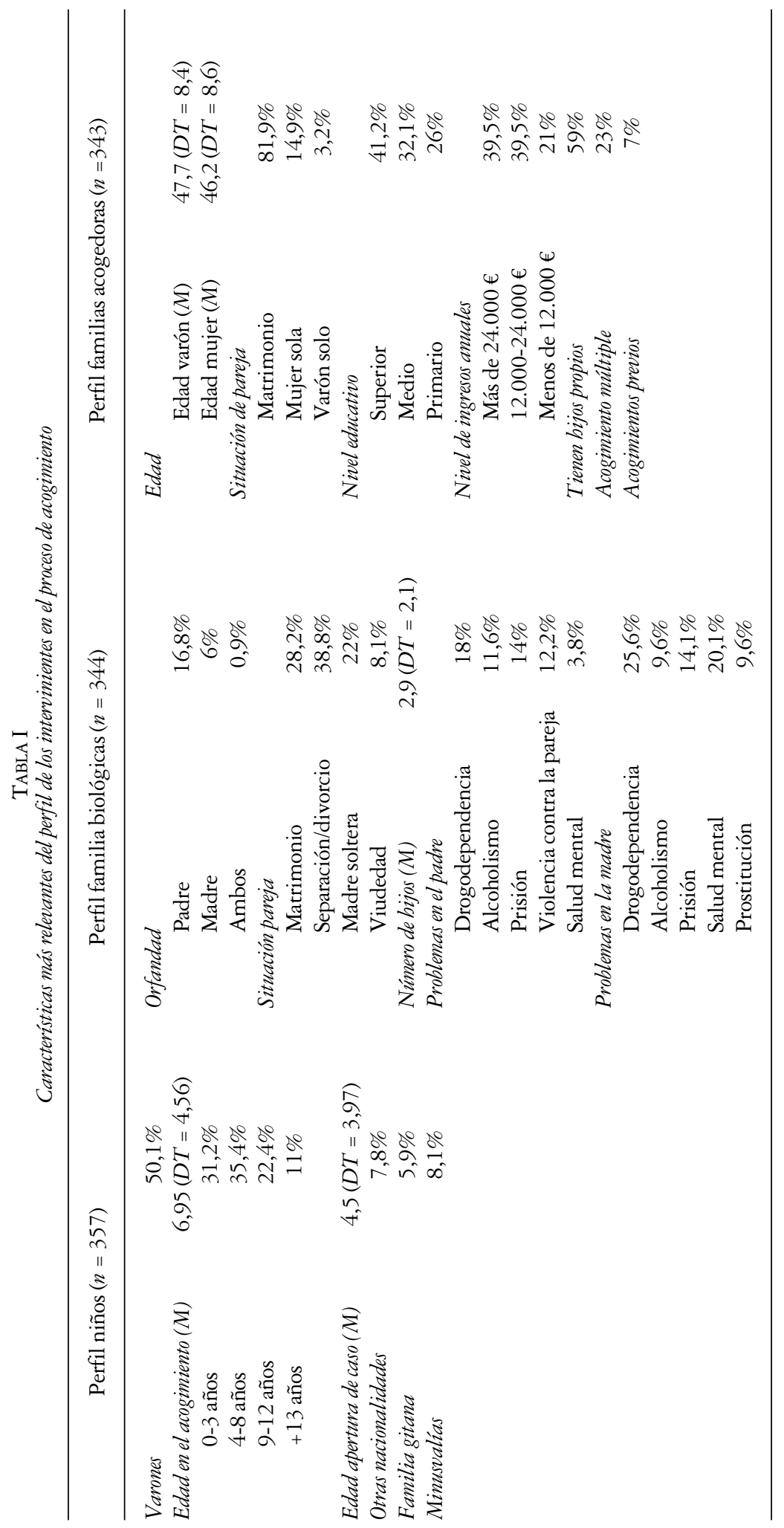


ron principalmente las drogodependencias (el 18\% de los padres y el 25,6\% de las madres), el alcoholismo (un 11,6\% de padres y 9,5\% de madres), así como la estancia en prisión de los progenitores ( $14 \%$ en ambos). En el caso de la madre también aparecieron de modo remarcable los problemas de salud mental $(20,1 \%)$ y prostitución $(9,6 \%)$.

Para el estudio de las familias acogedoras se trabajó con una muestra menor que la de niños y niñas, al coincidir algunos de ellos acogidos en la misma familia. La muestra es en este caso de 343 familias acogedoras.

En la inmensa mayoría se trató de parejas casadas (82\%). La media de edad de los acogedores se encontró entre los 46-47 años, con un rango entre los 25 y los 79, concentrándose la mitad de los casos en la edad intermedia entre 41-50 años. Respecto al nivel educativo presentaron estudios superiores un $41,2 \%$ y estudios medios, un $32,1 \%$. La situación laboral más común fue que los dos miembros de la familia trabajasen (64\%) y en torno al $40 \%$ ingresaban $24.000 €$ al año o más.

Más de la mitad de las familias (59\%) tenía hijos propios (en un 4,1\% eran adoptados) y la media fue de 2,1 hijos por familia (calculada solamente sobre las que tenían hijos). La edad de los hijos propios en relación al acogido fue mayor en el $77 \%$ de los casos, como se suele recomendar en los criterios para este tipo de situaciones, fue menor en un $11 \%$ y en el restante $12 \%$ tenían algún hijo mayor y otro menor.

El 23\% de las familias estaba realizando un acogimiento múltiple (más de un niño al mismo tiempo). Finalmente, se encontraron muy pocas ocasiones en que los acogedores hubieran realizado otro acogimiento previamente (7\%). La media de años que llevaban siendo acogedores fue de 5,1 años $(D T=3,5)$.

\section{El Proceso de Acogimiento}

El 82,7\% de los niños y niñas había tenido algún tipo de intervención anterior desde los servicios sociales de familia e infancia (Tabla II). Aproximadamente dos de cada tres casos habían pasado por un acogimiento residencial (69\%), en un $16 \%$ se había regularizado la situación tras un acogimiento informal por los mismos acogedores, y un 15,1\% provenían de otro acogimiento familiar. En estos casos se trató mayoritariamente de rupturas, es decir, de acogimientos interrumpidos por algún motivo ajeno a la consecución de objetivos, siendo la familia acogedora la que solicitó la interrupción del acogimiento en el $64 \%$ de las situaciones. Destacaron, entre los múltiples motivos, los referidos a los problemas de conducta del niño o niña y la enfermedad o indisposición de los acogedores. Cabe destacar que de estos niños y niñas con acogimientos previos, sólo una quinta parte había pasado por dos familias y únicamente un caso había experimentado el máximo de tres acogimientos anteriores. Un indicador importante es la necesidad de intervención judicial (lo que ocurre cuando los padres se oponen judicialmente a la medida administrativa de acogimiento) que se observó en el $46 \%$ de los casos.

En la intervención con infancia desprotegida los técnicos de los servicios elaboran un "plan de caso" que se diseña con la finalidad de establecer un objetivo principal de la intervención protectora. En los casos de separación del niño de su familia biológica, habitualmente puede ser: el retorno a la familia (cuando se prevé que los padres puedan recuperar las funciones de parentalidad); la integración definitiva en una nueva familia (mediante adopción o acogimiento); y la independencia (para los jóvenes sin posibilidad de permanecer en familia y que tendrán que independizarse). En los resultados obtenidos aparecieron algunas categorías adicionales, como la de "continuidad" (para expresar que el caso no tiene otra finalidad prevista que la permanencia en el acogimiento), y la de "estudio" referida a la situación de encontrarse en un proceso de evaluación inicial. Precisamente la primera de estas categorías, la de continuidad, es la que supuso más de dos terceras partes de los casos $(67 \%)$. Apareció a gran distancia el plan del retorno familiar (13\%), el plan de independencia (10\%), y finalmente la adopción $(4,7 \%)$ y el estudio $(4,7 \%)$. 
TABLA II

Características más relevantes del proceso del acogimiento

$\begin{array}{lc}\text { Intervenciones anteriores } & \\ \text { Acogimiento residencial } & 69,5 \% \\ \text { Acogimiento familiar } & 15,1 \% \\ \text { Intervención familiar } & 18,2 \% \\ & \\ \text { Con intervención judicial } & 46,5 \% \\ \text { Plan de caso } & \\ \text { Retorno a su familia } & 13,1 \% \\ \text { Adopción } & 4,7 \% \\ \text { Hasta mayoría de edad } & 10,2 \% \\ \text { Continuidad } & 67,4 \% \\ \text { Estudio } & 4,7 \% \\ & \\ \text { Existen visitas } & 64,1 \% \\ \text { Frecuencia de las visitas } & \\ \text { Semanal o quincenal } & \\ \text { Mensual } & 25,2 \% \\ & 50 \% \\ \text { Lugar de las visitas } & \\ \text { Punto de encuentro } & \\ \text { Lugares públicos } & 38,5 \% \\ \text { Hogar familia biológica } & 32,8 \% \\ \text { Hogar de los acogedores } & 18,2 \% \\ \end{array}$

Con respecto al porcentaje de casos con visitas de la familia de origen, casi dos tercios de los niños las recibían (64\%), siendo la madre la persona que las realizaba con más frecuencia $(47 \%)$. La periodicidad de las visitas más frecuente resultó ser la mensual (la mitad de los casos), mientras que el contacto semanal sólo representó el $13 \%$ de los casos. El lugar donde se producían las visitas con más frecuencia $(38,5 \%)$ fue el punto de encuentro o programas similares. Es de destacar que las visitas acontecían en lugares públicos como parques, centros comerciales o cafeterías en un 32,8\%. Las visitas requerían supervisión en un 36\% de los casos, de los que en un 93,3\% la supervisión la realizaban los profesionales de los servicios de infancia.

\section{Evaluación de resultados}

Los resultados se analizaron sobre una submuestra de 179 casos ya cerrados. La duración media de estos acogimientos fue de 3,41 años, constituyendo los de duración inferior a un año un 25,6\% y los más prolongados (más de siete años) un 12,5\%.

Se distinguieron tres grandes razones para poner fin al acogimiento familiar: el cumplimiento de la mayoría de edad del niño o niña acogido; la ruptura o interrupción prematura del acogimiento; y la decisión de cambio de medida por parte de los profesionales de los equipos de protección infantil (por haber cumplido los objetivos del acogimiento y poder regresar al hogar, o bien por ser posible una alternativa más favorable).

La decisión profesional de cambio de medida protectora fue el motivo principal de la finalización del acogimiento en casi la mitad de la muestra (48,6\%). Un dato especialmente relevante es que las rupturas se dieron en uno de cada cuatro casos (26\%). Por último, los casos que finalizaron el acogimiento debido a alcanzar la mayoría de edad fueron del 25,4\%, existiendo diez situaciones en las que no se pudo esclarecer el motivo del cierre.

No se encontraron diferencias por razón de sexo para la razón del cierre. En cambio, la edad de los niños y niñas en el momento de inicio del acogimiento sí arrojó 
diferencias estadísticamente significativas $\left[\chi^{2}(6,169)=75,05, p=.000\right]$, ya que predominaron los motivos de decisión de cambio hasta los ocho años, mientras que a partir de los nueve lo más frecuente fue la mayoría de edad (Tabla III). En los niños menores de tres años el cierre fue debido a una decisión técnica en un 85,7\% de los casos y en un 12,5\% por rupturas. Para el grupo de 4-8 años la decisión profesional de cambio de medida fue el motivo de cierre para más de la mitad de los casos $(60,5 \%)$, aunque las rupturas supusieron ya casi una tercera parte $(30,2 \%)$. El grupo de 9-12 años presentó la tasa de ruptura más alta con más de una tercera parte $(37 \%)$, pasando a ser el motivo más destacado la mayoría de edad $(45,7 \%)$, con algunos cambios por decisión técnica $(17,4 \%)$. Finalmente, en el grupo de los adolescentes se observó que más de la mitad de los casos que iniciaron el acogimiento con más de trece años cumplieron la mayoría de edad con la familia acogedora $(56,3 \%)$, presentando también pocos ceses por decisión de cambio $(18,8 \%)$ y un porcentaje de rupturas del $25 \%$.

TABLA III

Motivo de cierre según edad al inicio del acogimiento

\begin{tabular}{|c|c|c|c|c|c|c|c|c|c|c|}
\hline \multirow[b]{3}{*}{ MOTIVO DE CIERRE } & \multirow{2}{*}{\multicolumn{2}{|c|}{$0-3$}} & \multirow{2}{*}{\multicolumn{2}{|c|}{$4-8$}} & \multicolumn{2}{|c|}{ EDAD } & \multirow{2}{*}{\multicolumn{2}{|c|}{+13}} & \multirow{2}{*}{\multicolumn{2}{|c|}{ TOTAL }} \\
\hline & & & & & & & & & & \\
\hline & $n$ & $\%$ & $n$ & $\%$ & $n$ & $\%$ & $n$ & $\%$ & $n$ & $\%$ \\
\hline Decisión de cambio & 42 & 85,7 & 26 & 60,5 & 8 & 17,4 & 6 & 18,8 & 82 & 48,6 \\
\hline Mayoría de edad & 0 & 0 & 4 & 9,3 & 21 & 45,7 & 18 & 56,3 & 43 & 25,4 \\
\hline Ruptura & 6 & 12,5 & 13 & 30,2 & 17 & 37,0 & 8 & 25 & 44 & 26 \\
\hline TOTAL & 48 & 100 & 43 & 100 & 46 & 100 & 32 & 100 & 169 & 100 \\
\hline
\end{tabular}

Diferencias significativas $p=0.000$

La siguiente variable sobre el resultado hace referencia a la situación tras la finalización, lo que podríamos denominar el desenlace del acogimiento. Una vez cerrado el caso de acogimiento se observa que un 24,6\% volvió a un acogimiento residencial (coincidente en gran parte con las rupturas), y otro $23,4 \%$ fue adoptado por los propios acogedores. Únicamente un $18 \%$ tuvo un retorno familiar y un 16,2\% permaneció con los acogedores tras el cese de la medida. Ahora bien, como el tipo de desenlace tras el cese del acogimiento está íntimamente ligado al motivo de cierre, a continuación se detallan los resultados teniendo en cuenta ambos aspectos a la vez (Tabla IV).

TABLA IV

Situación tras el fin del acogimiento según el motivo de cese

\begin{tabular}{lllllll}
\hline & \multicolumn{9}{c}{$\begin{array}{c}\text { Motivo de cese } \\
\text { Ruptura } \\
\text { Situación }\end{array}$} & $n$ & $\%$ & $n$ & $\%$ & \multicolumn{2}{c}{$\begin{array}{c}\text { Mayoría de edad } \\
\end{array}$} & 9 & 11,1 & 32 & 71,4 & - & - \\
\hline Acogimiento residencial & 22 & 27,2 & 4 & 9,5 & 3 & 8,1 \\
Retorno familiar & - & - & - & - & 28 & 64,9 \\
Permanencia acogedores & 35 & 42 & 2 & 4,8 & 6 & 13,5 \\
Adopción acogedores & 3 & 3,7 & 1 & 2,4 & - & - \\
Acogimiento extensa & 8 & 9,9 & 2 & 4,8 & - & - \\
Adopción otra familia & 2 & 2,5 & 3 & 7,1 & - & - \\
Nuevo acogimiento ajena & - & - & - & - & 6 & 13,5 \\
Independencia & 3 & 3,6 & - & - & - & - \\
Otros & 82 & 100 & 44 & 100 & 43 & 100 \\
\hline Total & & & & & & \\
\hline
\end{tabular}


Cuando el motivo de cese se debió a cumplir 18 años, una parte importante de los jóvenes se quedó a vivir con sus acogedores $(64,9 \%)$. Además, a este último porcentaje podríamos añadir otro $13,5 \%$ que fue adoptado por estos acogedores tras la mayoría de edad, lo que supone en realidad un 78,4\% de continuidad en el hogar de acogida para los que cumplieron la mayoría de edad en él.

En el caso de cierre por ruptura, lo más habitual es que pasaran a un acogimiento residencial $(71,4 \%)$, aunque algunos regresaron con su familia $(9,5 \%)$ o pasaron a ser acogidos con una nueva familia $(9,5 \%)$.

Por último, cuando el cese del acogimiento se produjo por una decisión técnica de cambio de medida, la más frecuente consistió en la adopción por parte de los acogedores $(42 \%)$. El retorno familiar se produjo en algo más de la cuarta parte de estas decisiones $(27,2 \%)$ y a ello cabe sumar otro $10 \%$ de adopciones por otras familias diferentes de la acogedora, lo que indica que la mitad de las decisiones técnicas de cambio en acogimientos son para pasar a adopción $(51,9 \%)$.

\section{Discusión y conclusiones}

En España, a pesar de las reformas legales de los años ochenta y noventa que impulsaron la priorización del acogimiento en familia ajena frente al residencial, no se ha alcanzado el desarrollo que se esperaba, y esta medida sigue ocupando el tercer lugar por lo que se refiere a recurso sustitutivo, muy por detrás del acogimiento residencial y el acogimiento en familia extensa. Con los datos expuestos sobre el perfil de los niños acogidos, las familias biológicas y los acogedores, el proceso del acogimiento y la evaluación de resultados, se pretende contribuir a identificar algunos factores que pueden haber dificultado su desarrollo.

Referente a la edad de los niños al inicio del acogimiento, en la revisión realizada por Berridge y Cleaver (1987) y en Triseliotis, Borland y Hill (2000) se sitúa en torno a los cuatro años para los casos del Reino Unido, mientras que en nuestro estudio se eleva hasta los siete, probablemente debido al retraso entre la apertura del caso y la realización del acogimiento familiar. Efectivamente, la relación entre edad en el acogimiento y edad en la apertura del caso permite concluir que se tarda un promedio de más de dos años desde que se abre el expediente hasta que se realiza el acogimiento, tiempo que, como hemos visto, la mayoría tienen que permanecer en acogimiento residencial. En este sentido, un estudio realizado recientemente en Cataluña (Montserrat, 2008) muestra que, si se comparan los diferentes recursos, cuando los niños son propuestos para un acogimiento en familia extensa, en la mayoría de las ocasiones $(96,5 \%)$ se produce de manera inmediata; de los que son propuestos para acogimiento residencial, acaban ingresando rápidamente un $80 \%$; pero cuando se propone una medida de acogimiento en familia ajena, sólo llega a asignarse este recurso en la mitad de los casos (53\%), fundamentalmente debido a la ausencia de familias acogedoras. Mejorar los procesos de captación para aumentar el número de familias de acogida, continua siendo una asignatura pendiente.

Con respecto al perfil de las familias biológicas, existe un alto grado de rupturas familiares, y una alta incidencia de drogodependencias, tal y como se ha puesto de manifiesto en otros estudios españoles (Palacios y Jiménez, 2007). Coincidimos también con O’Donnell (1999) en que los padres están a menudo más ausentes del proceso, fallecidos, en paradero desconocido, desentendidos de sus hijos, etc. La alta tasa de drogodependencias, especialmente en la madre, no sólo es característica de este tipo de familias, sino que es también un factor que indica un difícil pronóstico para la reunificación familiar (Maluccio y Ainsworth, 2003; Terling, 1999), lo que podría incidir en la larga permanencia de estos niños con las familias de acogida.

En cuanto a los acogedores, nuestros hallazgos están en la línea de lo ya conocido en otras investigaciones, en las que se concluye que suele existir un buen nivel de estudios y de situación económica (Amorós et al., 2003; Ehrle y Geen, 2002), algo que contrasta 
enormemente con los acogedores en familia extensa, la mayoría abuelos, con bajo nivel de estudios y escasez de recursos económicos (Del Valle et al., 2002; Farmer y Moyers, 2008; Montserrat, 2007).

Por lo que respecta a las visitas de la familia biológica, sólo un $13 \%$ de los progenitores visita semanalmente a sus hijos, y la mitad acostumbran a hacerlo mensualmente, lo que enlaza con el bajo porcentaje de reunificación familiar. En este sentido, sorprende el dato de que el 35,9\% de los niños no reciben ninguna visita por parte de su familia biológica y en cambio se encuentran en acogimiento y no en adopción, cuestión que invita a la reflexión sobre la adecuación de la medida.

Los niños que son acogidos en familia provienen en su mayoría de un acogimiento residencial y en muy pocos casos $(15 \%)$ de otro acogimiento familiar. Estos datos contrastan enormemente con lo que ocurre en otros países donde los numerosos cambios de familia de acogida son un motivo de gran preocupación (Bullock, Courtney, Parker, Sinclair y Thoburn, 2006; Sinclair, Baker, Lee y Gibbs, 2007; Ward et al., 2005). Los acogimientos en nuestro país no presentan este problema de inestabilidad, en términos de cambios frecuentes de una familia a otra.

Esta estabilidad tiene que ver seguramente con la alta permanencia que presentan los acogimientos estudiados, donde se ha podido apreciar que el plan de caso más frecuente es el de "continuidad", es decir, que la finalidad del caso es permanecer en el acogimiento para dos terceras partes de los casos $(67 \%)$, seguida a gran distancia por el plan del retorno familiar (13\%). Este es uno de los resultados más preocupantes, ya que parece que el acogimiento familiar se utiliza como un recurso en sí mismo, como antes sucedía con el acogimiento residencial, donde una vez ubicado el menor no parecen existir esfuerzos para buscar una alternativa más definitiva (reunificación o adopción, fundamentalmente). Como consecuencia, hemos visto que existe un importante número de niños y niñas que alcanzan la mayoría de edad en situación de acogimiento familiar.

En el estudio de los resultados evaluados mediante casos cerrados, la tasa de ruptura alcanza a uno de cada cuatro casos. Este grado de ruptura se encuentra en línea con otros trabajos internacionales (Minty, 1999; Sallnäs et al., 2004), aunque las variaciones son muy grandes entre países e investigaciones. La consecuencia de esta ruptura del acogimiento es fundamentalmente la vuelta a un acogimiento residencial, lo que establece una nueva e importante diferencia con otros países donde la ruptura de un acogimiento familiar supone casi siempre intentar otro nuevo (Berrick et al., 1998; Frame et al., 2000). Aunque las rupturas afectan a adolescentes fundamentalmente (la mitad de ellas se producen en esta edad) se ha podido comprobar que estas rupturas afectan con mayor frecuencia a menores que habían sido acogidos en la franja de 9-12 años, que a los acogidos siendo ya adolescentes. Este dato debe relacionarse con la importancia de realizar adecuados seguimientos, especialmente en la transición a la adolescencia, para ofrecer apoyos y orientación a las familias acogedoras.

Es importante señalar también que se da un elevado número de adopciones por parte de las familias acogedoras, un dato muy inesperado si se parte de la clara diferencia que debe existir entre el perfil de adoptantes y acogedores. No obstante, debe matizarse que estas adopciones se han producido en una de las comunidades autónomas en concreto, donde ello se permite (e incluso parece alentarse). En cambio, en el resto de las comunidades de la muestra la adopción por acogedores sólo se acepta de manera excepcional. Esta pluralidad de criterios ante la posibilidad de la adopción por parte de los acogedores es una muestra de la gran variabilidad que existe entre las administraciones en la práctica del acogimiento familiar. Como ya se concluyó en un informe previo (Del Valle y Bravo, 2003) esta disparidad de criterios afecta también a cuestiones tan sustanciales como lo que se entiende por acogimiento simple y permanente, o la existencia de compensación económica a los acogedores y su cuantía. Se trata, a nuestro juicio, de aspectos demasiado importantes, incluso algunos definidos inequívocamente en nuestro ordena- 
miento jurídico (como la diferencia entre simple y permanente), como para que se utilicen de manera tan dispar.

Parece claro que tras veinte años de desarrollo, el acogimiento familiar presenta unas características muy particulares en España. La escasa implantación del acogimiento en familia ajena supone que los niños y niñas sin familiares dispuestos a acogerlos van a tener muy escasas posibilidades de disfrutar de un hogar de acogida y van a tener que seguir utilizando servicios de atención residencial, los cuales siguen suponiendo una red muy amplia y en crecimiento (Bravo y Del Valle, 2009). Algunos de los resultados observados pueden ayudar a encontrar explicaciones a esta baja implantación. Probablemente la gran estabilidad y permanencia que muestran los datos de nuestro estudio, donde los niños acaban siendo adoptados, o se quedan a vivir con mucha facilidad tras cumplir la mayoría de edad, hace que estos acogedores no estén disponibles para nuevos acogimientos, y que su ciclo de acogida se reduzca a un solo caso, al igual que puede ocurrir fácilmente con los acogedores que sufren una ruptura. Por unos u otros motivos, lo cierto es que de las 343 familias acogedoras de nuestra muestra sólo un $7 \%$ estaba repitiendo la experiencia y, dada la enorme dificultad de captar familias para este fin, el hecho de que no realicen más de una acogida dificulta enormemente la expansión que esta medida precisa.

Todo indica que en España se sigue una línea de acogimientos más bien permanentes (aunque sin llegar a definirlos expresamente así), algunos de ellos quizá para evitar la dificultad de los procesos que requieren las adopciones, y en otros casos probablemente sean la consecuencia de la falta (o escasa efectividad) de programas de reunificación que trabajen con las familias biológicas.

Los resultados de este estudio apuntan importantes retos de futuro. Por un lado, debemos mejorar el nivel de intervención con las familias biológicas, tanto para prevenir la separación como para conseguir la reintegración familiar tras una medida de separación. En segundo lugar, se deben aunar criterios entre administraciones para la definición del acogimiento familiar y sus objetivos básicos, especialmente la diferencia entre acogimiento y adopción, así como su posible relación excluyente o no. Pero lo más importante, a nuestro juicio, es el reto de cómo conjugar el mantenimiento de esta estabilidad y permanencia, que es tan primordial para los niños y niñas acogidos, con la necesidad de que las familias acogedoras no se consuman en una única experiencia y puedan desarrollar varias acogidas consecutivas. Así se conseguiría ampliar el número de niños que disfrutan de esta posibilidad e incrementaría el conjunto de familias acogedoras con larga trayectoria y experiencia, facilitando la efectividad de esta medida.

En cuanto a las limitaciones de este trabajo, se trata de un estudio descriptivo cuyo valor radica en presentar por primera vez este tipo de datos en España. Que un trabajo tan descriptivo sea relevante, es un claro indicador del estado tan precario de nuestra investigación en este tema. El hecho de realizarse sobre seis de las diecisiete comunidades autónomas plantea inevitables problemas de generalización, y aunque estas seis comunidades pueden representar aceptablemente la práctica nacional en su conjunto (constituyen el 75\% de los acogimientos en España), el grado en que los resultados puedan ser válidos para otra comunidad concreta es dudoso, fundamentalmente debido a la variabilidad de criterios y prácticas entre ellas. Es de esperar que nuevas investigaciones emerjan desde una perspectiva más inferencial, contribuyendo a desentrañar los factores que expliquen aspectos tan cruciales como las rupturas o los criterios de éxito de las reunificaciones.

\section{Referencias}

Amorós, P., Palacios, J., Fuentes, N., LeÓn, E. \& Mesas, A. (Eds.) (2002). Programa para la formación de familias acogedoras de urgencia y diagnóstico. Barcelona: Fundación La Caixa.

Amorós, P., PAlacios, J., Fuentes, N., LeÓn, E. \& Mesas, A. (Eds.) (2003). Familias Canguro: Una experiencia de protección a la infancia. Barcelona: Fundación La Caixa. 
Bernedo, I., Fuentes, M. J. \& FernándeZ, M. (2008). Behavioral problems in adolescents raised by their grandparents. The Spanish Journal of Psychology, 11 (2), 453-463

BeRRICK, J. D., NEEDELL, B., BARTH, R. \& JONHSON-REID, M. (1998). The tender years: Toward developmentally sensitive child welfare services for very young children. Nueva York: Oxford University Press.

Berridge, D. \&. Cleaver, H. (Ed.) (1987). Foster Home Breakdown. Oxford: Basil Blackwell.

Bravo, A. \& Del Valle, J. F. (2009). Crisis y revisión del acogimiento residencial: su papel en la protección infantil. Papeles del Psicólogo, 30 (1), 42-52.

Bullock, R., Courtney, M. E., Parker, R., Sinclair, I. \& Thoburn, J. (2006). Can the corporate state parent? Children and Youth Services Review, 28 (11), 1344-1358.

Dei Valle, J. F., AlvareZ-BaZ, E. \& Bravo, A. (2002). Acogimiento en familia extensa. Perfil descriptivo y evaluación de necesidades en una muestra del Principado de Asturias. Bienestar y Protección Infantil, 1 (1), 34-56.

Del Valle, J. F., Álvarez-Baz, E. \& Bravo, A. (2003). Evaluación de resultados a largo plazo en acogimiento residencial de protección a la infancia Infancia y Aprendizaje, 26 (2), 531-542.

Del Valle, J. F. \& Bravo, A. (2003). La situación del acogimiento familiar en España. Madrid: Ministerio de Trabajo y Asuntos Sociales. Disponible en: http://www.mepsyd.es/observatoriodeinfancia/documentos/Informe_acogida_familiar.pdf

EHRLE, J. \& GEeN, R. (2002). Kin and non-kin foster care: Findings from a national survey. Children and Youth Services Review, $24(1), 15-35$

FaRMER, E. \& MOYERs, S. (2008). Kinship Care. Fostering Effective Family and Friends Placements. Londres: Jessica Kingsley.

FrAme, L., BerRick, J. D. \& BRODOWSKY, M. L. (2000). Understanding reentry to out-of-home care for reunified infants. Child Welfare League of America, 79 (4), 339-369.

LEY 21/1987, de 11 de noviembre, por la que se modifican determinados artículos del Código Civil y de la Ley de Enjuiciamiento Civil en materia de adopción y otras formas de protección de menores. (B.O.E. n 275, de 17 de noviembre de 1987).

Lumbreras, H., Fuentes, M. J. \& Bernedo, I. (2005). Perfil descriptivo de los acogimientos con familia extensa de la provincia de Málaga. Revista de Psicología Social Aplicada, 15 (1-2), 93-117.

Maluccio, A. N. \& Ainsworth, F. (2003). Drug use by parents: A challenge for family reunification practice. Children $\mathcal{E}$ Youth Services Review, 25 (7), 511-533.

MinISTERIO DE TRABAJO Y ASUNTOS SOCIALES (2007). Estadistica básica de protección a la infancia, $n^{\circ} 9$. Madrid: Ministerio de Trabajo y Asuntos Sociales.

MinTy, B. (1999). Annotation: Outcomes in long-term foster family care. Journal of Child Psychology and Psychiatry, 40 (7), 991-999.

Molero, R., Mora, M. J., Albiñana, P. \& Gimeno, A. (2006). El acogimiento en familia extensa: un estudio del perfil y las necesidades. Valencia: Ayuntamiento de Valencia.

Montserrat, C. (2007). Kinship foster care: A study from the perspective of the caregivers, the children and the child welfare workers. Psychology in Spain, 11 (1), $42-52$.

Montserrat, C. (2008). Niños, niñas y adolescentes acogidos por sus familiares: ¿qué sabemos, qué conocemos? Barcelona: Generalitat de Catalunya.

MONTSERRAT, C. \& CASAS, F. (2006). Kinship foster care from the perspective of quality of life: Research on the satisfaction of the stakeholders. Applied Research in Quality of Life, 1 (3), 227-237.

O`DonNell, J. M. (1999). Involvement of African American fathers in kinship care services Social Work, 44 (5), 428-441

Palacios, J. \& AMORós, P. (2006). Recent Changes in Adoption and Fostering in Spain. British Journal of Social Work, 36 (6), 921-935.

PALACIOS, J. \& JimÉnEZ, J. M. (2007). El acogimiento familiar en Andalucía. Sevilla: Consejería para la Igualdad y Bienestar Social de la Junta de Andalucía. Disponible en: http://www.juntadeandalucia.es/observatoriodelainfancia/oia/ esp/descargar.aspx? id $=1602 \&$ tipo $=$ documento.

Sallnäs, M., Vinnerljung, B. \& Westermark, P. K. (2004). Breakdown of teenage placements in Swedish foster and residential care. Child E Family Social Work, 9 (2), 141-152.

SinCLAIR, I. (2005). Fostering Now. Messages from Research. Londres: Jessica Kingsley.

Sinclair, I., BAKer, C., LeE, J. \& GIBBS, I. (2007). The Pursuit of Permanency. A Study of the English Child Care System. Londres: Jessica Kingsley.

SinClair, I., BAKER, C., WILSON, K. \& GIBBS, I. (2005). Foster Children. Where they go and How they Get On. Londres: Jessica Kingsley.

Sinclair, I., GibBS, I. \&WILSON, K. (2004). Foster Carers: Why They Stay and Why They Leave. Londres: Jessica Kingsley.

TERLING, T. (1999). The efficacy of family reunification practices: Reentry rates and correlates of reentry for abused and neglected children reunited with their families. Child Abuse and Neglect, 23 (12), 1359-1370.

Triseliotis, J., Borland, M. \& Hill, M. (Eds.) (2000). Delivering foster care. Londres: BAAF.

VANDERfaeillie, J., VAN Holen, F. \& Coussens, S. (2008). Why foster care placements breakdown? A study into the factors influencing foster care placement breakdown in Flanders. En C. Canali, T. Vecchiato \& J. K. Whittaker (Eds.), Assessing the "Evidence-base" of Intervention for Vulnerable Children and Their Families (pp. 77-79). Padova: Fondazione Emanuela Zancan.

W ARD, H., Skuse, T. \& MunRo, E. R. (2005). "The best of times, the worst of times". Young people views of care and accommodation. Adoption and Fostering, 29 (1), 8-17. 